\title{
Estimation of acoustic impedance, it's excess value and molar sound velocity of the binary mixture of 2-Butanol and m-Xylene for different compositions at different temperatures
}

\author{
Md. Sydur Rahman*, Muhammad Habibullah \\ Department of Chemistry, World University of Bangladesh \\ * corresponding author : sydur.rahman@science.wub.edu.bd \\ DOI : 10.20885/ijca.vol3.iss1.art1
}

\begin{tabular}{l} 
ARTICLE INFO \\
\hline Received : 20 December 2019 \\
Revised : 02 February 2020 \\
Published : 15 March 2020 \\
Keywords : acoustic impedance, \\
physico-acoustic, butanol, xylene
\end{tabular}

\begin{abstract}
Measurements of physico-acoustic properties at an ultrasonic level have been studied intensively for the binary mixture of 2-Butanol $+\mathrm{m}$-Xylene system as a function of the composition at $298.15 \mathrm{~K}$ to $323.15 \mathrm{~K}$ by $5 \mathrm{~K}$ intervals and at atmospheric pressure. Acoustic impedance $(Z)$, excess acoustic impedance $\left(Z^{\mathrm{E}}\right)$ and molar sound velocity $(\mathrm{R})$ have been evaluated from the experimental values of speeds of sound and densities. The variation of these properties with composition and temperature suggests the presence of dipoleinduced dipole interactions, hydrogen bond, induced electrostatic and dispersion forces. The observed negative values of $Z^{\mathrm{E}}$ over the entire composition range of the system reinforce significant interactions between unlike molecules that dominate over other types. The variation of molar sound velocity $(R)$ of the entire mixture suggests the rarefaction of the liquid mixture under study.
\end{abstract}

\section{INTRODUCTION}

From the very earlier time, the scientists and the physical chemists show greater interest in the term of mixture properties. A mixture is nothing but the interaction of different molecules. So the appearance of some new phenomena different from their individual pure states may be observed. By studying the liquid-liquid mixture at different mole fractions, the trend of changing of properties can find out. The temperature also does a strong effect on this change when liquids are mixed together; therefore, there may be either contraction or expansion in volume and thus deviating from the additive rule $[1,2,3,4]$. Still, today liquids have been studied extensively by both theoretical and experimental techniques using various physicochemical properties. Knowledge of ultrasonic velocity and density of different mole fractions of solvent-solute mixtures can further be used to determine the acoustic impedance, excess acoustic impedance and molar sound velocity. Sound generally travels through materials under the influence of sound pressure. As molecules or atoms of a compound are bound elastically to each other, the excess pressure results in a wave propagating through the compound. Among the sound properties, acoustic impedance is important i. in determining the acoustic transmission and reflection at the boundary of two materials having different acoustic impedances, ii. In designing ultrasonic transducers and iii. In assessing absorption of sound in a medium. Molar sound velocity, on the other hand, provides a clear idea on the intermolecular interactions occurred in any liquid mixture.

Alcohols, in particular, 2-Butanol (or 2-Bu-OH) is an organic compound normally found as an equal mixture of the two stereoisomers [5], a racemic mixture of (R)-(-)-2-Bu-OH and (S)-(+)-2$\mathrm{Bu}-\mathrm{OH}$. This secondary alcohol is a flammable, colourless liquid that is soluble in water and completely miscible with polar solvents such as ethers and other alcohols [6,7], m-Xylene (or m$\mathrm{Xln}$ ) is an aromatic hydrocarbon, based on benzene with two methyl substituents. Generally, xylene is used as solvent $\&$ is most widely used as a clearing agent $[8,9]$. $\mathrm{m}-\mathrm{X} \ln$ is a common component of ink, rubber, and adhesive and In thinning paints and varnishes [10,11]. In the petroleum industry, $\mathrm{m}$ $\mathrm{Xln}$ is also a frequent component of paraffin solvents. It is also used as a raw material in the manufacture of 2,4- and 2,6-xylidine as well as a range of smaller-volume chemicals. 
One most important application of these two liquids is both of them are frequently used in the pharmaceutical sector as residual solvents in the formulation of various essential drugs. So it is urgent to evaluate the mixing properties of this binary mixture to estimate the nature of mixing. The systematic survey of the literature shows that there is no systematic study especially on acoustic properties (acoustic impedance, molar sound velocity) present in the binary mixture containing 2$\mathrm{Bu}-\mathrm{OH}$ and $\mathrm{m}-\mathrm{Xln}$ for every specific proportion along with the temperature. In view of this importance, it is of interest to study the physico-acoustic properties to estimate their acoustic nature in the mixture along with identifying the trend of changing for different molecular composition very intensively to understand the molecular interactions between them.

\section{EXPERIMENTAL}

The chemical reagents employed were of analytical graded and were obtained from Sigma Aldrich, England, with a minimum purity of $99.5 \%$. The water used in all experimental works was double distilled. The binary mixtures of $2-\mathrm{Bu}-\mathrm{OH}$ and $\mathrm{m}$-Xln were prepared by using an analytical balance with a precision of $\pm 0.1 \mu \mathrm{g}$ and later were converted to the different components of the mixture using the dilution method. Special care was taken to prevent evaporation and the introduction of moisture into the experimental samples.

The density and speed of sound of all binary mixtures, including pure solvents, were measured using an oscillatory densimeter (Anton Paar DSA 5000). The temperature was automatically controlled with an uncertainty of $\pm 0.01 \mathrm{~K}$. The apparatus was calibrated frequently by measuring the double-distilled water and dry air. The uncertainty of density for all the solvent systems studied is less than $\pm 0.00005 \mathrm{gcm}^{-3}$, and the uncertainty of speed of sound is less than $\pm 1 \mathrm{~ms}^{-1}$.

\section{RESULTS AND DISCCUSION}

The density values of binary mixtures, including $\mathrm{m}$-Xln as a function of the mole fraction of 2$\mathrm{Bu}-\mathrm{OH}(\mathrm{x} 1)$ at temperatures $(298.15$ to 323.15$) \mathrm{K}$ are listed in Table 1 . It is found that the density decreases as the temperature increases as well as with the increasing proportion of 2-Bu-OH. Corresponding speeds of sound are shown in Table 2. Here the existence of weak electrostatic force between the interacting molecules changes their structures. The structural changes affect the compressibility, and hence there is a sharp change in ultrasonic velocity. It is noticed regarding $\mathrm{u}$ at all temperatures in the order: $\mathrm{m}-\mathrm{Xln}>2-\mathrm{Bu}-\mathrm{OH}$ and with the addition of 2-Bu-OH, u decreases slowly for 2-Bu-OH $+\mathrm{m}-\mathrm{Xln}$ system (Table 2 ).

TABEL I. Density of 2-Bu-OH + m-Xln system for different molar ratios at $298.15 \mathrm{~K}$ to $323.15 \mathrm{~K}$ by $5 \mathrm{~K}$ intervals

\begin{tabular}{|c|c|c|c|c|c|c|c|}
\hline \multicolumn{2}{|c|}{$\begin{array}{l}\text { Mole fraction of } \\
\mathrm{X}\end{array}$} & \multicolumn{6}{|c|}{$\begin{array}{c}\text { Experimental Density }(\rho) \\
\times 10^{3}\left(\mathbf{k g ~ m}^{3}\right)\end{array}$} \\
\hline $\begin{array}{c}2-\mathrm{Bu}- \\
\mathrm{OH} \\
x_{1} \\
\end{array}$ & $\begin{array}{c}\mathbf{m}-X \ln \\
\boldsymbol{x}_{2}\end{array}$ & 298.15 K & 303.15 K & $308.15 \mathrm{~K}$ & 313.15 K & $318.15 \mathrm{~K}$ & $\begin{array}{c}323.15 \\
K\end{array}$ \\
\hline 0.0000 & 1.0000 & 0.8599 & 0.8555 & 0.8512 & 0.8469 & 0.8425 & 0.8381 \\
\hline 0.0500 & 0.9500 & 0.8569 & 0.8525 & 0.8481 & 0.8437 & 0.8392 & 0.8348 \\
\hline 0.1007 & 0.8994 & 0.8542 & 0.8497 & 0.8453 & 0.8408 & 0.8363 & 0.8318 \\
\hline 0.1499 & 0.8501 & 0.8516 & 0.8471 & 0.8426 & 0.8381 & 0.8336 & 0.8290 \\
\hline 0.1998 & 0.8002 & 0.8486 & 0.8442 & 0.8396 & 0.8351 & 0.8305 & 0.8259 \\
\hline 0.2496 & 0.7504 & 0.8460 & 0.8415 & 0.8369 & 0.8324 & 0.8278 & 0.8231 \\
\hline 0.2998 & 0.7002 & 0.8433 & 0.8388 & 0.8343 & 0.8297 & 0.8251 & 0.8204 \\
\hline
\end{tabular}




\begin{tabular}{llllllll}
\hline 0.3498 & 0.6502 & 0.8407 & 0.8362 & 0.8316 & 0.8270 & 0.8223 & 0.8177 \\
0.3996 & 0.6004 & 0.8381 & 0.8335 & 0.8290 & 0.8243 & 0.8197 & 0.8150 \\
0.4498 & 0.5502 & 0.8354 & 0.8309 & 0.8263 & 0.8217 & 0.8170 & 0.8123 \\
0.4993 & 0.5008 & 0.8328 & 0.8282 & 0.8236 & 0.8190 & 0.8143 & 0.8096 \\
0.5490 & 0.4510 & 0.8300 & 0.8254 & 0.8209 & 0.8162 & 0.8115 & 0.8068 \\
0.5997 & 0.4003 & 0.8272 & 0.8226 & 0.8180 & 0.8134 & 0.8087 & 0.8040 \\
0.6493 & 0.3507 & 0.8246 & 0.8201 & 0.8155 & 0.8109 & 0.8062 & 0.8014 \\
0.7026 & 0.2974 & 0.8213 & 0.8168 & 0.8123 & 0.8076 & 0.8029 & 0.7982 \\
0.7497 & 0.2503 & 0.8185 & 0.8140 & 0.8095 & 0.8048 & 0.8002 & 0.7954 \\
0.8001 & 0.1999 & 0.8156 & 0.8112 & 0.8067 & 0.8021 & 0.7974 & 0.7927 \\
0.8483 & 0.1518 & 0.8128 & 0.8084 & 0.8039 & 0.7994 & 0.7947 & 0.7900 \\
0.9009 & 0.0991 & 0.8092 & 0.8049 & 0.8004 & 0.7959 & 0.7913 & 0.7866 \\
0.9503 & 0.0497 & 0.8059 & 0.8016 & 0.7973 & 0.7928 & 0.7882 & 0.7836 \\
1.0000 & 0.0000 & 0.8027 & 0.7985 & 0.7942 & 0.7898 & 0.7853 & 0.7807 \\
\hline
\end{tabular}

TABEL II. Sound Velocity of 2-Bu-OH + m-Xln system for different molar ratios at $298.15 \mathrm{~K}$ to $323.15 \mathrm{~K}$

\begin{tabular}{|c|c|c|c|c|c|c|c|}
\hline \multicolumn{2}{|c|}{$\begin{array}{c}\text { Mole fraction of } \\
\mathrm{X}\end{array}$} & \multicolumn{6}{|c|}{$\begin{array}{c}\text { Speed of sound }(\mathbf{u}) \\
(\mathrm{m} / \mathrm{s})\end{array}$} \\
\hline $\begin{array}{c}2-\mathrm{Bu}- \\
\mathbf{O H} \\
\mathbf{x}_{1}\end{array}$ & $\begin{array}{c}\mathbf{m}-\mathbf{X l n} \\
\mathbf{x}_{2}\end{array}$ & 298.15 K & $303.15 \mathrm{~K}$ & 308.15 K & $313.15 \mathrm{~K}$ & 318.15 K & $\begin{array}{c}323.15 \\
\mathbf{K}\end{array}$ \\
\hline 0.0000 & 1.0000 & 1317.99 & 1297.32 & 1276.82 & 1256.52 & 1236.44 & 1216.99 \\
\hline 0.0500 & 0.9500 & 1308.42 & 1288.19 & 1268.11 & 1248.16 & 1228.37 & 1209.08 \\
\hline 0.1007 & 0.8994 & 1299.39 & 1279.25 & 1259.31 & 1239.53 & 1219.92 & 1200.89 \\
\hline 0.1499 & 0.8501 & 1292.12 & 1272.15 & 1252.15 & 1232.44 & 1212.90 & 1193.94 \\
\hline 0.1998 & 0.8002 & 1285.32 & 1265.32 & 1245.51 & 1225.85 & 1206.37 & 1187.45 \\
\hline 0.2496 & 0.7504 & 1279.04 & 1259.12 & 1239.37 & 1219.77 & 1200.34 & 1181.48 \\
\hline 0.2998 & 0.7002 & 1273.24 & 1253.37 & 1233.67 & 1214.12 & 1194.74 & 1175.93 \\
\hline 0.3498 & 0.6502 & 1267.81 & 1248.00 & 1228.33 & 1208.84 & 1189.50 & 1170.71 \\
\hline 0.3996 & 0.6004 & 1262.79 & 1243.04 & 1223.75 & 1203.89 & 1184.57 & 1165.80 \\
\hline 0.4498 & 0.5502 & 1258.00 & 1238.25 & 1218.66 & 1199.21 & 1179.91 & 1161.17 \\
\hline 0.4993 & 0.5008 & 1253.42 & 1233.68 & 1214.12 & 1194.68 & 1175.40 & 1156.65 \\
\hline
\end{tabular}




\begin{tabular}{llllllll}
\hline 0.5490 & 0.4510 & 1248.84 & 1229.14 & 1209.61 & 1190.20 & 1170.92 & 1152.19 \\
0.5997 & 0.4003 & 1244.46 & 1224.84 & 1205.34 & 1185.95 & 1166.70 & 1147.93 \\
0.6493 & 0.3507 & 1240.90 & 1221.35 & 1201.90 & 1182.56 & 1163.32 & 1144.62 \\
0.7026 & 0.2974 & 1236.32 & 1216.86 & 1197.48 & 1178.17 & 1158.96 & 1140.24 \\
0.7497 & 0.2503 & 1232.60 & 1213.26 & 1193.88 & 1174.62 & 1155.43 & 1136.74 \\
0.8001 & 0.1999 & 1228.92 & 1209.69 & 1190.53 & 1171.39 & 1152.30 & 1133.68 \\
0.8483 & 0.1518 & 1225.11 & 1206.13 & 1187.16 & 1168.20 & 1149.33 & 1130.75 \\
0.9009 & 0.0991 & 1220.34 & 1201.56 & 1182.76 & 1163.93 & 1145.09 & 1126.65 \\
0.9503 & 0.0497 & 1215.98 & 1197.49 & 1178.97 & 1160.38 & 1141.73 & 1123.46 \\
1.0000 & 0.0000 & 1211.83 & 1193.70 & 1175.57 & 1157.15 & 1138.84 & 1120.79 \\
\hline
\end{tabular}

The acoustic impedance of a material is the displacement of the medium's particles by sound energy. It is one of the basic parameters to understand the intrinsic and elastic properties of the medium. Acoustic impedance $(Z)$ and excess values of acoustic impedance $\left(Z^{\mathrm{E}}\right)$ are estimated by using Eq. $(1,2)[12,13$,$] .$

$$
\begin{aligned}
& \mathrm{Z}=\rho \mathrm{u} \\
& \mathrm{Z}^{\mathrm{E}}=\mathrm{Z}-\left(\mathrm{X}_{1} \mathrm{Z}_{1}+\mathrm{X}_{2} \mathrm{Z}_{2}\right)
\end{aligned}
$$

Experimentally obtained values of excess properties $\left(\mathrm{Z}^{\mathrm{E}}\right)$ for this system can be fitted by a least square method using Redlich-Kister Eq. (3) [14]:

$$
\begin{aligned}
& (\mathrm{Y})^{\mathrm{E}}=\mathrm{x}_{1} \mathrm{x}_{2} \sum_{i \geq 0}\left(1-2 \mathrm{x}_{1}\right) \mathrm{B}_{i} \\
& \mathrm{R}=\mathrm{VU}^{1 / 3}
\end{aligned}
$$

Where, $\mathrm{x}_{1}, \mathrm{x}_{2}$ is the mole fraction of component 1 and 2 and Bi the coefficient of Redlich-Kister equation. Another very important parameter is molar sound velocity which was calculated following Eq. (4) [15]. The estimated values of $Z$ in the full range of composition $0 \leq x 1 \leq 1$ between 298.15 $\mathrm{K}$ and $323.15 \mathrm{~K}$ are graphically represented by Figure 1 . As acoustic impedance is one of the critical parameters that can be used to understand the intrinsic and elastic properties of the medium so this decreasing of $\mathrm{Z}$ values with the increasing proportion of 2-Bu-OH suggests that the existence of significant molecular interaction gradually increases. The estimated values of $Z^{\mathrm{E}}$ in the full range of composition of the system are represented in Figure 2.

By examining Figure $(1,2)$ the following observations are noticed regarding $Z$ and $Z^{\mathrm{E}}$

1. At all temperatures, $\mathrm{Z}$ of the pure liquids varies in the order: $m-\mathrm{Xln}>2-\mathrm{Bu}-\mathrm{OH}$.

2. With the addition of 2-Bu-OH, $\mathrm{Z}$ decreases almost linearly to reach the value of pure alcohol.

3. $Z^{\mathrm{E}}$ values are negative over the whole range of composition within the studied range of temperatures.

4. As the alcohol concentration raises the negative $Z^{\mathrm{E}}$ values for both systems increases sharply until they reach the pick of the curve, after which decrease slowly toward pure alcohol.

5. The negative maxima of $\mathrm{Z}^{\mathrm{E}}$ for $2-\mathrm{Bu}-\mathrm{OH}+\mathrm{m}-\mathrm{X} \ln$ system found at $\mathrm{x}_{1} \approx 0.35$. 


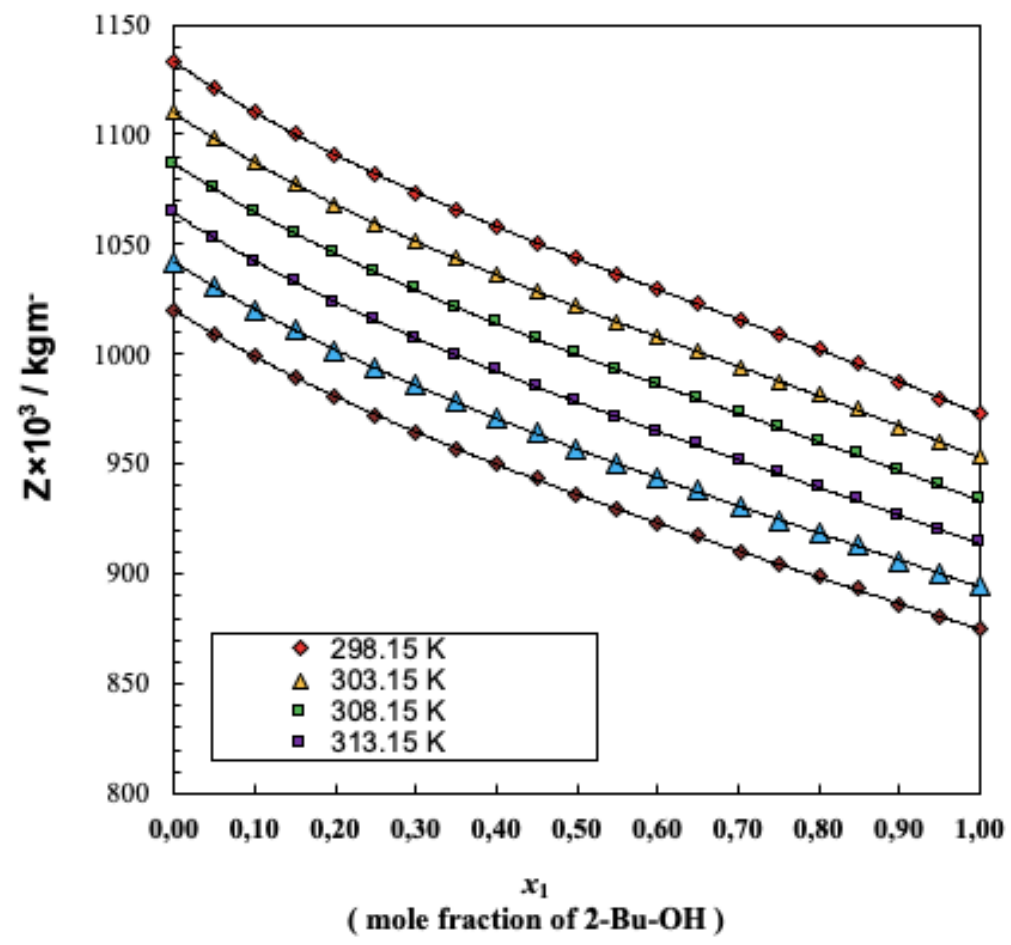

Figure 1. Acoustic impedance curve for different mole fractions at different temperatures. The graph presents the linear decreasing trend that indicates the significant interactions between the mixture components in a much-disciplined way.

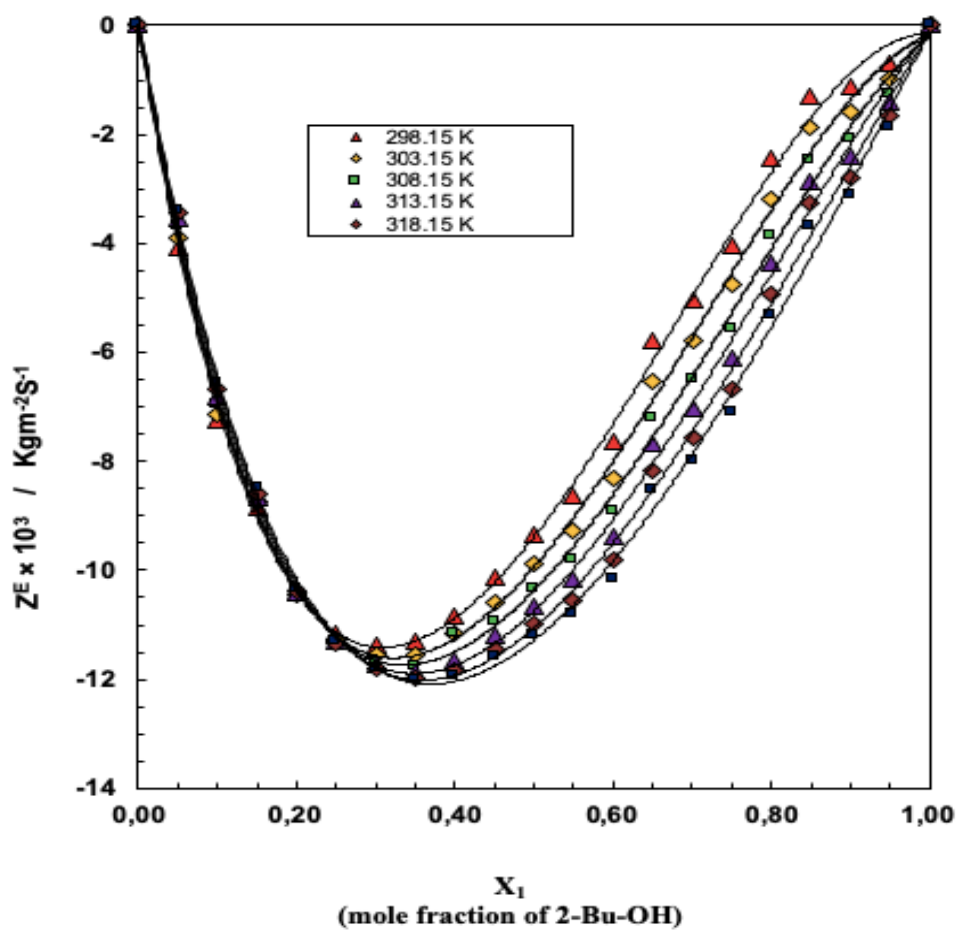

Figure 2. Excess acoustic impedance curve for different mole fractions at different temperatures. Negative excess values indicate the compactness due to molecular rearrangement and the extent of molecular interactions in liquid mixtures. 
Here $\mathrm{Z}$ of $\mathrm{m}-\mathrm{Xln}>2-\mathrm{Bu}-\mathrm{OH}$ and thus there is almost a linear decrease of $\mathrm{Z}$ for the binary mixture with the increasing mole fraction of $2-\mathrm{Bu}-\mathrm{OH}$. Negative excess values play a vital role in assessing the compactness due to molecular rearrangement and the extent of molecular interactions in liquid mixtures. The specific attraction between these unlike molecules is for physical intermolecular forces including electrostatic and induction forces between a permanent dipole and an induced dipole. Thus structural characteristics of the component arise from the regular fitting of one component into other structure due to the differences in shape and size of the components. They were breaking hydrogen bonds of adjacent alcohol molecules also responsible for negative excess values which also indicate strong molecular interaction between the, unlike molecules.

TABLE III. Molar sound velocity of 2-Bu-OH $+\mathrm{m}-\mathrm{X} \ln$ system for different molar ratios at different temperatures

\begin{tabular}{|c|c|c|c|c|c|c|c|}
\hline \multicolumn{2}{|c|}{$\begin{array}{c}\text { Mole fraction of } \\
\text { X }\end{array}$} & \multicolumn{6}{|c|}{$\begin{array}{l}\text { Molar sound velocity (Rao's Constant } \mathrm{R} \text { ) of } 2-\mathrm{Bu}-\mathrm{OH}+\mathrm{m}-\mathrm{X} \text { In system } \\
\text { for different molar ratios at different temperatures }\left(\mathrm{m}^{2} / \mathrm{mol}\right)(\mathrm{m} / \mathbf{s})^{1 / 3}\end{array}$} \\
\hline \multirow{2}{*}{$\begin{array}{c}\text { 2-Bu- } \\
\mathbf{O H} \\
\mathbf{x}_{1}\end{array}$} & \multirow{2}{*}{$\begin{array}{c}\mathbf{m}-X \mathbf{l n} \\
\mathbf{x}_{2}\end{array}$} & & & & & & \\
\hline & & $298.15 \mathrm{~K}$ & 303.15 K & $308.15 \mathrm{~K}$ & 313.15 K & $318.15 \mathrm{~K}$ & 323.15 K \\
\hline 0.0000 & 1.0000 & 1353.6370 & 1353.3280 & 1353.0190 & 1352.7150 & 1352.4330 & 1352.3070 \\
\hline 0.0500 & 0.9500 & 1334.5770 & 1334.5080 & 1334.4340 & 1334.3570 & 1334.2790 & 1334.3110 \\
\hline 0.1007 & 0.8994 & 1315.0390 & 1315 & 1315 & 1315.1410 & 1315 & 1315.4400 \\
\hline 0.1499 & 0.8501 & 1296.3450 & 1296.4380 & 1296.4970 & 1296.6160 & 1296.7630 & 1297.0700 \\
\hline 0.1998 & 0.8002 & 1278.0930 & 1278.1940 & 1278.3340 & 1278 & 7100 & 1279.0850 \\
\hline 0.2496 & 0.7504 & 1259.5400 & 1259.6760 & 1259.8530 & 1260.0600 & 1260.3120 & 1260.7340 \\
\hline 0.2998 & 0.7002 & 1240.9260 & 1241.0820 & 1241.2830 & 1241.5190 & 1241.8050 & 1242.2650 \\
\hline 0.3498 & 0.6502 & 1222.4340 & 1222.6150 & 1222.8310 & 1223.0970 & 1223.4090 & 1223.8950 \\
\hline 0.3996 & 0.6004 & 1204.0830 & 1204.2780 & 1204.6130 & 1204.7770 & 1205.1070 & 1205.6080 \\
\hline 0.4498 & 0.5502 & 1185.5840 & 1185.7880 & 1186.0370 & 1186.3320 & 1186.6790 & 1187.2090 \\
\hline 0.4993 & 0.5008 & 1167.3850 & 1167.5880 & 1167.8480 & 1168.1510 & 1168.5130 & 1169.0470 \\
\hline 0.5490 & 0.4510 & 1149.1860 & 1149.3960 & 1149.6630 & 1149.9770 & 1150.3430 & 1150.8900 \\
\hline 0.5997 & 0.4003 & 1130.6220 & 1130.8440 & 1131.1150 & 1131.4310 & 1131.8080 & 1132.3460 \\
\hline 0.6493 & 0.3507 & 1112.3450 & 1112.5700 & 1112.8420 & 1113.1660 & 1113.5460 & 1114.1060 \\
\hline 0.7026 & 0.2974 & 1093.0670 & 1093.2940 & 1093.5710 & 1093.8930 & 1094.2730 & 1094.8270 \\
\hline
\end{tabular}




\begin{tabular}{llllllllll}
\hline 0.7497 & 0.2503 & 1075.9850 & 1076.2140 & 1076.4740 & 1076.7960 & 1077.1750 & 1077.7310 \\
0.8001 & 0.1999 & 1057.4670 & 1057.6900 & 1057.9690 & 1058.2910 & 1058.6700 & 1059.2270 \\
0.8483 & 0.1518 & 1039.7570 & 1039.9920 & 1040.2750 & 1040.6040 & 1041.0100 & 1041.5430 \\
0.9009 & 0.0991 & 1020.7530 & 1020.9770 & 1021.2500 & 1021.5650 & 1021.9380 & 1022.4790 \\
0.9503 & 0.0497 & 1002.7090 & 1002.9310 & 1003.2020 & 1003.5190 & 1003.8880 & 1004.4240 \\
1.0000 & 0.0000 & 984.4760 & 984.7010 & 984.9890 & 985.2700 & 985.6580 & 986.1860 \\
\hline
\end{tabular}

Molar sound velocity or Rao's constant (R) gives a clear idea about the extent of interactions taking place between the molecules of this system. It indicates whether there are associative or dissociative interaction occurs. The variation of molar sound velocity listed in Table 3 shows that $R$ is not constant for different molar ratios, so the interactions of this mixture are expected to be associative. However, the decreasing trend of the molar sound velocity with the increasing mole fraction of 2-Bu-OH suggests that the rarefaction of the medium.

\section{CONCLUSIONS}

The purpose of this work is to evaluate the acoustical impedance and molar sound velocity of this binary mixture and thus to determine how sound energy correlates through molecular levels of this mixture and how the intermolecular interactions of it change. Acoustic impedance is an essential characteristic of materials for nearly all sonic and ultrasonic applications. In the electrical analogy, it determines the efficiency of every transmission in the system from one element to another. In this study, the experimental densities and speed of sound of 2-Bu-OH, m-Xln and their binary system have been measured at temperatures $(298.15$ to 323.15$) \mathrm{K}$ and in atmospheric pressure. Excess acoustical impedance $\left(\mathrm{Z}^{\mathrm{E}}\right)$ of this binary mixture was calculated and fitted with the Redlich-Kister equation. From these parameters, we got clear ideas about valuable acoustic properties as well as about the existing interactions between these mixing components, which are mainly for dipoleinduced dipole interaction, induced electrostatic and dispersion force. The observed negative excess values $\mathrm{ZE}$ indicate the significant interaction between the different molecules, whereas $\mathrm{R}$ values suggest that the overall mixing is associative.

\section{References}

[1] R. M. Arvind and R. M. Sunil," Excess Molar Volumes and Viscosities for the Binary Mixtures of nOctane, n-Decane, n-Dodecane, and n-Tetradecane with Octane-2-ol at 298.15 K," J Thermodyn, vol. 11, 2013

[2] A. B. Sawant, M. Hasan, V. J. and Naukudkar," Excess Volume and Deviation in Viscosities of Binary Mixtures of o-Dichlorobenzene and o-Chlorophenol with Ethyl Methyl Ketone, Methyl Isobutyl Ketone, Propiophenone and Cyclohexane," Chem Sci Trans, vol. 2, 263-269, 2013.

[3] D. de Carvalho, V. L. Lanchote, P. S. Bonato, R. H. C. Queiroz, and A. C. Santos," Dreossi S A C. A new derivatization procedure for the analysis of hippuric acid and $m$-methyl-hippuric acid by gas chromatography," Int Arch Occup Env Hlth, vol. 63, 33-37, 1991

[4] D. D. Ezekiel, M. N. Simphiwe, A. I. David, and E. E. Eno," Density, Dynamic Viscosity and Derived Properties of Binary Mixtures of Methanol, Ethanol, n-Propanol, and n-Butanol with Pyridine at T = (293.15, 303.15, 313.15 and 323.15)," Int J Electrochem Sci., vol. 7, 11101 - 11122, 2012

[5] W. Shi, B. A. Ncev, S. Bhat, and J. O. Liu," Impact of Absolute Stereochemistry on the Antiangiogenic and Antifungal Activities of Itraconazole," ACS Med Chem Lett vol. 1, 155-159, 2010 
[6] The national institute for occupational safety and health(NIOSH), http://www.cdc.gov/niosh/index.htm

[7] F. Frank, H. Howard, W. M. Thomas, A. Frookson, P. Pomerantz, and D. B. Brooks," Preparation and Physical Properties of Several Aliphatic Hydrocarbons and Intermediates," J Res Nation Bur Stand, vol. $38,1779,1947$

[8] R. Kandyala, S. P. C. Raghavendra, and S. T. Rajasekharan,"Xylene: An overview of its health hazards and preventive measures," J Oral Maxillofac Pathol, vol 14, 1-5, 2010

[9] W. Kunhua, F. Chuming, L. Tao, Y. Yanmei, Y. Xin, Z. Xiaoming, G. Xuezhong , and L. Xun,”A novel non-toxic Xylene substitute(SBO) for histology," Afr J Tradit Complem Altern Med., vol 9, 43-49, 2012

[10] J. Palau, J. M. Penya-Roja, C. Gabaldon, F. J. Alvarez-Hornos, F. Sempere, and V. Martinez-Soria,’UV photocatalytic oxidation of paint solvent compounds in air using an annular $\mathrm{TiO}_{2}$-supported reactor," $\mathrm{J}$ Chem Tech Biotech, vol. 86, 273-281, 2011

[11] N. Satou, K. Ishihara, M. Hiratsuka, H. Tanaka, Y. Endo, S. Saito, Y. Iwakura, W. Warren, N. Leonard,Hirasawa,'Induction of Thymic Stromal Lymphopoietin Production by Xylene and Exacerbation of Picryl Chloride-Induced Allergic Inflammation in Mice," Int Arch Allergy Immunol, vol. 157, 194-201, 2012.

[12] G. R. Balaji, G. G. Naganathan, and G. Veerapandian,"Acoustic and volumetric studies of benzophenone with alcihils and toluene at 303K and atmospheric pressure," Int J Tech Res Appl., vol. 37, 52-59, 2016

[13] M. V. Rathnam, K. R. Bhanushali, R.T. Sayed, and M. S. S. Kumar,'Densities, viscosities and speeds of sound for binary liquid mixtures of hexyl acetate and $\mathrm{PhX}\left(\mathrm{X}=\mathrm{F}, \mathrm{CL}, \mathrm{Br}, \mathrm{NO}_{2}\right)$ compounds at different temperatures," Eur Chem Bull. Vol. 2, 434-444, 2011

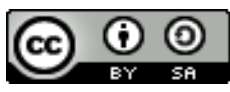

Jurnal IJCA is licensed under aÂ Creative Commons Attribution ShareAlike 4.0 